Article

\title{
Local External/Internal Symmetry of Smooth Manifolds and Lack of Tovariance in Physics
}

\author{
Torsten Asselmeyer-Maluga ${ }^{1,2}$ and Jerzy Król 2,3,*(1) \\ 1 German Aerospace Center (DLR), Rosa-Luxemburg-Str. 2, 10178 Berlin, Germany; \\ Torsten.Asselmeyer-Maluga@dlr.de \\ 2 Copernicus Center for Interdisciplinary Studies ul. Szczepańska 1/5, 31-011 Cracow, Poland \\ 3 Cognitive Science and Mathematical Modelling Chair, University of Information Technology and \\ Management, ul. Sucharskiego 2, 35-225 Rzeszów, Poland \\ * Correspondence: iriking@wp.pl
}

Received: 1 October 2019; Accepted: 18 November 2019; Published: 20 November 2019

check for updates

\begin{abstract}
Category theory allows one to treat logic and set theory as internal to certain categories. What is internal to SET is 2-valued logic with classical Zermelo-Fraenkel set theory, while for general toposes it is typically intuitionistic logic and set theory. We extend symmetries of smooth manifolds with atlases defined in Set towards atlases with some of their local maps in a topos $\mathcal{T}$. In the case of the Basel topos and $\mathbb{R}^{4}$, the local invariance with respect to the corresponding atlases implies exotic smoothness on $\mathbb{R}^{4}$. The smoothness structures do not refer directly to Casson handless or handle decompositions, which may be potentially useful for describing the so far merely putative exotic $\mathbb{R}^{4}$ underlying an exotic $S^{4}$ (should it exist). The tovariance principle claims that (physical) theories should be invariant with respect to the choice of topos with natural numbers object and geometric morphisms changing the toposes. We show that the local $\mathcal{T}$-invariance breaks tovariance even in the weaker sense.
\end{abstract}

Keywords: exotic $R^{4}$; smooth toposes; tovariance; symmetry and categories in physics

\section{Introduction}

Symmetry, and patterns of breaking it, are indisputably one of the basic guiding principles in modern natural science. The development of classical and quantum physics, in particular, can be seen as exemplifying the invariances of theories with respect to global or local symmetry groups. The gauge groups of quantum field theory, and diffeomorphism groups of smooth manifolds as in general relativity (GR), are intrinsic concepts underlying our understanding of the world. Along with symmetries, the patterns of symmetry breaking have become equally important. 20th-century mathematics has given us an alternative competing view as to its foundations. Basically, this was achieved via the development of category theory, in which we can view the modifications of set theory, logic, and geometry in a unified way. Such as approach shed light on new understanding of symmetry and physics. This is particularly the case in respect of topos theory, which has been found to be applicable to physics to a degree that could hardly have been envisaged (e.g., [1-9]). Along with clarifying the role of category theory in relation to the foundations of mathematics and quantum physics, researchers became interested in an emerging fundamental symmetry of a new kind, namely the invariance of theories with respect to the choice of new foundations of mathematics given by the broad class of toposes. The class of toposes contains ones with natural numbers objects with geometric morphisms as morphisms of toposes [10]. Later, the invariance of a theory with respect to the choice of such toposes came to be named "tovariance", and this has served to indicate its conceptual resemblance to GR covariance [11,12]. 
In the case of geometric morphisms between toposes and a theory formulated in the geometric language, the invariance of the theory with respect to the choice of a topos with NNO has a direct solution: The invariant theory just is the theory in such language. So, one takes a theory in the intuitionistic logic formulated in geometric language and this theory will be invariant without any modifications in any topos with NNO. All its interpretations in toposes with NNO survive the modifications by geometric morphisms of toposes. Moreover, this kind of global symmetry will not affect a Set description, provided it is limited to intuitionistic and geometric means. The proposal put forward by Landsman et all claims that such invariance (tovariance) should be the guiding principle for every physical observation: i.e., that one cannot decide experimentally which topos with NNO is actually better to use compared to any other. Such a democracy of toposes would show that one can freely switch between toposes, while invariant physics remains as the geometric and intuitionistic content of a theory. In addition, this, in turn, would have a quite surprising consequence: Any quantum theory would be equivalent to classical one in a special topos [11].

In the present paper, we study smoothness structures on manifolds which allow for certain local modifications by smooth toposes (e.g., [13]). Our position is that the tovariance principle is an example of global symmetry and the global invariance of a theory requires (intuitionistic) geometric language. The passage from global to local symmetry has become very fruitful in physics and mathematics. The local gauge symmetry generates additional invariant physical fields in in such theories as those of electrodynamics or the standard model of particles and fields in general. Guided by this kind of role played by local symmetry in physics, we apply a similar strategy to smooth manifolds and the invariance of their smoothness structures under modifications by toposes with NNO. "Local," on this approach, means defined on a local chart from the smooth atlas on a manifold $M$. Thus, to locally modify $M$ by a smooth topos means considering certain of its local charts internally in a topos. The resulting invariant smoothness structure of such a construction must be modified. Choosing the Basel topos $\mathcal{B}$ extensively studied by Moerdijk and Reyes in [13] as a locally deforming smooth topos, and given $M=\mathbb{R}^{4}$, the shift of the smoothness structure forces one to replace the standard $\mathbb{R}^{4}$ by an exotic $R^{4}$. Subsequent local modifications of $R^{4}$ gives rise also to a certain exotic $R^{4}$ nondiffeomorphic to the initial one. However, we do not know at present whether the sequence of such modified smoothings leads to a set of pairwise different and nondiffeomorphic $R^{4}$ 's.

The motivations behind developing such a description of exotic smoothness on $R^{4}$ come from both from physics and mathematics. First, we do not know any analytical forms of smooth functions on an exotic $R^{4}$, nor do we know any Riemann metric on it. This is particularly important in physics, where exotic $R^{4}$ s are becoming increasingly applicable to crucial problems often in contexts where there is no alternative option available. A pertinent example is the recent proof by Etesi [14] that large

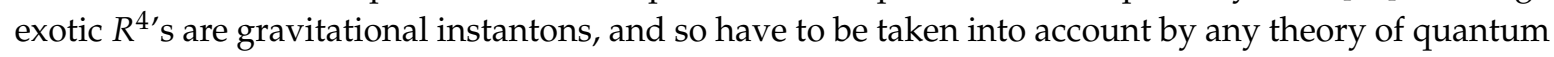
gravity with GR as its classical limit, or in the calculation of the realistic value of the cosmological constant which, moreover, is a topological invariant [15]. Knowing analytic expressions on an exotic $R^{4}$ would certainly shed light on the possible construction of quantum gravity and the cosmological constant problem. Second, we are seriously lacking in insights into the structure of large exotic $R^{4 \text { 's. }}$ The method of Casson handles or even handle decompositions, successfully explaining the structure

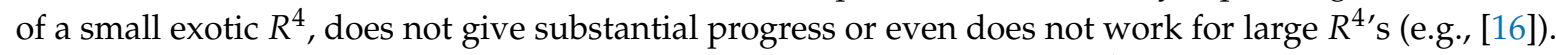
The proposing of an alternative mathematical construction for these $R^{4}$ 's a much-anticipated event. Finally, such an alternative description, valid in principle for an exotic $R^{4}$ allowing finitely many open covers in its smooth atlas, would be especially useful for understanding an exotic $R^{4}$ obtained from putative exotic 4-spheres. Such an $R^{4}$ would remain exotic after one-point compactification,

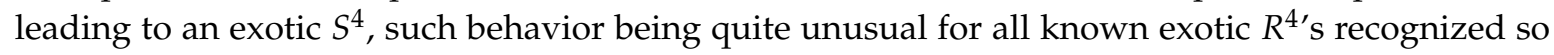
far. The approach in this paper is aimed at employing the extended symmetry principle for analyzing exotic smoothness structures on $\mathbb{R}^{4}$. The extension is two-fold: Topos-theoretic-it allows for internal domains in the Basel topos-and local-i.e., local on $M$-modifications. Even though the invariance of a theory with respect to the global symmetry of toposes (tovariance) forces the language of the theory 
to be geometric, in the case of local symmetry the invariance transcends geometric morphisms, as the object of natural numbers in the Basel topos has to be set as $N$ distinct from $\mathbb{N}$.

As a result, we must allow for twisted geometric morphisms between toposes deforming local atlases on $M$. We show that the invariance of such smooth manifolds with respect to the above 'change of toposes' traced in Set leads to a change of smoothness structure on $\mathbb{R}^{4}$. Juxtaposing this result with the successful physical application of exotic $R^{4}$ s indicates that the tovariance principle of Landsman et all is to be broken.

The main result of the paper is Theorem 5 which states that given any exotic $R^{4}$, and modifying its smoothness structure locally by $\mathcal{B}$, one gets (as invariant in Set) a new smoothness structure that is nondiffeomorphic to $R^{4}$. On this basis, we perform an analysis of the general tovariance principle which even in its weak form, is presumably broken up to the equivalence of exotic $R^{4}$ and Set-invariant local $\mathcal{B}$-structures on $\mathbb{R}^{4}$. Assuming this alleged equivalence, one obtains a statement along the lines of Conjecture 1. As far as we know, the approach presented in this paper is new, though it can be seen as essentially extending the results in [17-19].

\section{Results}

Let us recall that a topological $n$-dimensional manifold is a Hausdorff, metrizable topological space $M$ together with an atlas of charts $\left\{\left(U_{\alpha}, \phi_{\alpha}\right)\right\}_{\alpha \in I}$ where $M=\bigcup_{\alpha \in I} U_{\alpha}$ and the maps $\phi_{\alpha}: U_{\alpha} \rightarrow$ $\phi\left(U_{\alpha}\right) \subseteq \mathbb{R}^{n}$ are homeomorphisms onto their images being open sets of $\mathbb{R}^{n}$.

An atlas is smooth whenever for any pair of indices $\alpha, \beta \in I$ and $U_{\alpha \beta}=U_{\alpha} \cap U_{\beta} \neq \varnothing$ the maps

$$
\phi_{\alpha \beta}:=\left.\phi_{\beta} \circ \phi_{\alpha}^{-1}\right|_{\phi_{\alpha}\left(U_{\alpha \beta}\right)}: \phi_{\alpha}\left(U_{\alpha \beta}\right) \rightarrow \phi_{\beta}\left(U_{\alpha \beta}\right) .
$$

are smooth with smooth inverses $\left(\phi_{\alpha \beta}\right)^{-1}=\phi_{\beta \alpha}$. The manifold $M$ with such an atlas is a smooth manifold.

The smooth structure of a smooth manifold $M$ is defined as the maximal atlas $\left\{\left(U_{\alpha}, \phi_{\alpha}\right)\right\}_{\alpha \in I}$ of local smooth charts.

An open cover $\left\{\left(U_{\alpha}, \phi_{\alpha}\right)\right\}_{\alpha \in I}$ of a topological manifold $M$ is called a good cover if all the $U_{\alpha}$ and all their non-empty finite intersections are contractible topological spaces.

A smooth good open cover of a smooth $n$-manifold $M$ is a good cover whose all finite non-empty intersections are diffeomorphic to an open ball, hence to $\mathbb{R}^{n}$.

Lemma 1. Every smooth manifold admits a good smooth open cover.

Proof. The proof follows from the fact that every open cover of a smooth manifold admits a subordinated smooth division of unity [20] (Th. 2.25, p. 54) and that this last is equivalent to the paracompactness of $M$. However, each paracompact smooth manifold admits a smooth good open cover (for the proof see e.g., [21].)

So, from now on we can always assume that the cover of a smooth manifold we work with is open good. Let $\mathbb{M}$ be the category of smooth manifolds and smooth maps [13] and Set be the category of sets and functions. Set can be naturally seen as a topos

1. The terminal object 1 in Set is an arbitrary singleton since there exists a unique arrow (function) $X \rightarrow 1$ for every set $X$.

2. There exist exponentiable set $X^{Y}$ of functions for every pair $(X, Y)$ of sets;

3. The fibered products: Given $f: B \rightarrow A$ and $g: C \rightarrow A$ their fibered product is the set $B \times{ }_{A} C=$ $\{(b, c) \in B \times C: f(b)=g(c)\}$.

4. The sub-object classifier and truth object: $\Omega=\{0,1\}$ determines subsets as the codomain of the characteristic functions. The truth arrow reads $1 \stackrel{\mathrm{T}}{\rightarrow} \Omega$. 
2-valued classical logic is thus written in the $\Omega$ and the classical ZF theory of sets can be assigned to a well-pointed abelian toposes [21].

Let $\mathcal{T}$ be a Grothendieck topos which is a topos of sheaves on a site (e.g., [13,22]) with 1. a sub-object classifier as defined in the sheaves toposes, 2. the internal logic is now intuitionistic and internal reasoning is performed without any use of the axiom of choice in set theory and the excluded third low $(p \vee \neg p=1)$, 3. intuitionistic set theory. $\mathbb{M}$ is defined in Set but can be embedded to certain toposes e.g., smooth toposes widely analyzed in [13]. Let $\mathcal{B}$ be the Basel topos which is one of the smooth toposes defined in [13]. Then it holds

Lemma 2 ([13] Lemma 5.2, p. 286). The embedding of the category $\mathbb{M}$ into $\mathcal{B}, s: \mathbb{M} \hookrightarrow \mathcal{B}$, is full and faithful.

Remark 1. The topos $\mathcal{B}$ is a Grothendieck topos which is construed as the category of sheaves on the site $\mathbb{B}$. Then the global section functor applied to $\mathcal{B}, \Gamma: \mathcal{B} \rightarrow$ Set, has a left adjoint $\Delta$, i.e., the locally constant sheaf functor which is the sheafification of the constant presheaf in the presheaves category Set $\mathbb{B}^{\mathbb{B}^{\circ}}$

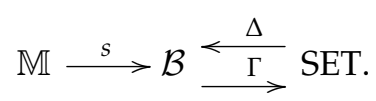

Thus, one can switch between Set and $\mathcal{B}$ by these two functors. As we will see later they create the unique geometric morphisms between $\mathcal{B}$ and Set (see Section 2.3).

Remark 2. The category $\mathbb{L}$ of loci is the opposite category of smooth rings and smooth maps between them [13]. The profound examples of smooth rings are $C^{\infty}\left(\mathbb{R}^{n}\right), n=1,2, \ldots$ and quotients of these spaces by ideals Id $\subset C^{\infty}\left(\mathbb{R}^{n}\right)$. $\mathbb{L}$ already contains all smooth manifolds from $\mathbb{M}$. The embedding $s: \mathbb{M} \rightarrow \mathbb{L}$ is depicted by the same latter $s$ (as to $\mathcal{B}$ ). Thus, smooth manifolds are replaced consistently in new categorical environment by smooth rings of functions.

Remark 3. We have direct description of $s: \mathbb{M} \rightarrow \mathbb{L}$. Let $\otimes_{\infty}$ be the coproduct in the category of smooth rings. Given any $M \in \mathbb{M}$ there exists $n \in \mathbb{N}$ and the ideal Id in $C^{\infty}\left(\mathbb{R}^{n}\right)$ such that

$$
\begin{array}{r}
s(M)=C^{\infty}(M)=C^{\infty}\left(\mathbb{R}^{n}\right) / I d \\
s(\mathbb{R})=C^{\infty}(\mathbb{R}) \text { and } s(M \times N)=s(M) \otimes_{\infty} s(N) \\
s\left(\mathbb{R}^{n}\right)=C^{\infty}\left(\mathbb{R}^{n}\right)=C^{\infty}(\mathbb{R}) \otimes_{\infty} \ldots \otimes_{\infty} C^{\infty}(\mathbb{R})=s(\mathbb{R}) \otimes_{\infty} \ldots \otimes_{\infty} s(\mathbb{R}) \\
\text { Additionally, for } s: \mathbb{M} \rightarrow \mathcal{B}: \forall_{M \in \mathbb{M}} \Gamma(s(M)) \stackrel{\text { diff }}{\simeq} M, \forall_{M, N \in \mathbb{M}} \Gamma\left(s(N)^{s(M)}\right) \stackrel{\text { diff }}{\simeq} C^{\infty}(M, N) .
\end{array}
$$

Let $C^{\infty}(M)$ in the category $\mathbb{L}$ (as opposite to smooth rings) be $\ell C^{\infty}(M)$ (the locus). It holds already for $\mathbb{L}$ [13] (Proposition 1.2, p. 60)

Proposition 1. The functor $s: \mathbb{M} \rightarrow \mathbb{L}$ given by $s(M)=\ell C^{\infty}(M)$, is full and faithful.

Remark 4. Given the topos Set $\mathbb{L}^{\text {op }}$ of presheaves on $\mathbb{L}$ one embeds $\mathbb{M}$ to Set $\mathbb{L}^{\mathbb{L}^{\text {op }}}$. This embedding is given by the Yoneda embedding which sends $M \in \mathbb{M}$ to the (representable) presheaf $\underline{M}$

$$
\underline{M}=\mathbb{L}(-, s(M))
$$

where $\mathbb{L}(-, s(M))$ is the class (set) of all morphisms in $\mathbb{L}$ ending at $s(M)$.

Remark 5. The site for the topos $\mathcal{B}$ is $\mathbb{B}$ which is just $\mathbb{L}$ being the category of loci $\mathbb{L}$ with the special Grothendieck topology [13] (pp. 285-286). It holds [13] (Lemma 5.2, p. 286).

Lemma 3. The Yoneda embedding $Y: \mathbb{L} \rightarrow$ Set $^{\mathbb{L}^{\text {op }}}$ factors through $\mathcal{B} \hookrightarrow \operatorname{Set}^{\mathbb{B}^{0 p}}$. 
Finally, $\mathbb{M} \ni M \rightarrow s(M)=\underline{M}:=\mathbb{B}(-, s(M))$ in $\mathcal{B}$.

In every Grothendieck topos with natural numbers object there exists the canonical representation of NNO given by the so-called constant sheaf $\underline{\mathbb{N}}=\Delta(\mathbb{N}):=\mathbb{N}$. This 'constant sheaf' is the sheaf corresponding to the constant presheaf $\mathbb{N}$. Moreover, given two toposes $\mathcal{T}_{1}$, $\mathcal{T}_{2}$ with the canonical constant sheaves NNO's $N_{1}, N_{2}$, and geometric morphisms $g: \mathcal{T}_{1} \rightarrow \mathcal{T}_{2}, g^{*} N_{1} \stackrel{\text { iso }}{=} N_{2}$, so thus these canonical NNO's are preserved by geometric morphisms.

However, there appear variety of difficulties when trying to do analysis on manifolds internally to $\mathcal{B}$ with respect to $\mathbb{N}$. In particular, [13] (p. 226)

- $\quad s: \mathbb{M} \rightarrow \mathcal{B}$ does not preserve compactness, e.g., $[0,1] \subset R=s(\mathbb{R})$ is not compact;

- $\quad s$ does not preserve open covers;

- $\quad s$ does not preserve partitions of unity subordinated to open covers;

- $\quad$ the $\operatorname{ring} R$ is not any local ring.

We will see later how to cure these unwanted properties.

\subsection{Local Ext/Int Symmetry on Smooth Manifolds}

Given a smooth $\mathbb{R}^{n}$ (in Set) and $s: \mathbb{M} \rightarrow \mathcal{B}$ sending $\mathbb{R}^{n}$ to $\mathcal{B}$ our present aim is to find descriptions of $s\left(\mathbb{R}^{4}\right)$ distinguishing its exotic smoothness structure from the standard one. This is well-defined question since the functor $s: \mathbb{M} \rightarrow \mathcal{B}$ sends fully and faithfully all smooth manifolds from Set internally to $\mathcal{B}$.

Remark 6. $R^{4}$ depicts any of nonstandard smoothings on $\mathbb{R}^{4}$. They are called exotic smooth $\mathbb{R}^{4}$, i.e., $R^{4 \text { 's }}$ (e.g., [16]). Each such smoothness structure of $\mathbb{R}^{4}$ is a smooth manifold homeomorphic to the topological $\mathbb{R}^{4}$ but nondiffeomorphic to it with the standard smoothing. The standard smooth structure of $\mathbb{R}^{n}$ is such that the product $\mathbb{R} \times \mathbb{R}^{n-1}$ is smooth. There is unique standard smooth $\mathbb{R}^{n}, n=1,2, \ldots$. It is known that there exist at least two families, each of cardinality of the continuum, of exotic $R^{4}$ 's: Small and large exotic $R^{4}$ 's. The later are not smoothly embeddable in the standard $\mathbb{R}^{4}$, or $S^{4}$, as open subsets while the small $R^{4}$ 's allow for such embedding.

Let $A$ be a smooth ring and $\ell A$ the corresponding locus in $\mathbb{L}$. We use abbreviation $\underline{\ell A}$ for the sheaf in $\mathcal{B}$ representing $A$, i.e., $s(A)=\underline{\ell A}$.

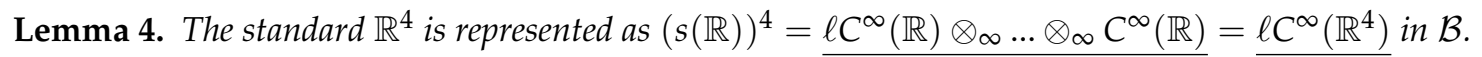

Proof. The standard smooth $\mathbb{R}^{4}$ in Set is the global smooth Cartesian product of 4 factors $\mathbb{R} \times \ldots \times \mathbb{R}$. It is sent to $C^{\infty}\left(\mathbb{R}^{4}\right)$ in $\mathbb{B}$. This last is $\ell C^{\infty}(\mathbb{R}) \otimes_{\infty} \ldots \otimes_{\infty} C^{\infty}(\mathbb{R})$ in $\mathbb{B}$ by (2) and (3). $\mathbb{L}$ is $\mathbb{B}$ with modified Grothendieck topology hence loci are objects in $\mathbb{B}$ and sheaves are representable (Remarks 4 and 5).

Thus, the standard smooth $\mathbb{R}^{4}$ from Set is $R^{4}=\underline{\ell C^{\infty}\left(\mathbb{R}^{4}\right)}$ in $\mathcal{B}$.

Lemma 5. Any small exotic $\mathbb{R}^{4}$ is represented in $\mathcal{B}$ as $\ell C^{\infty}\left(\mathbb{R}^{5}\right) /$ id and large exotic $\mathbb{R}^{4}$ by $\ell C^{\infty}\left(\mathbb{R}^{m}\right) / I d$

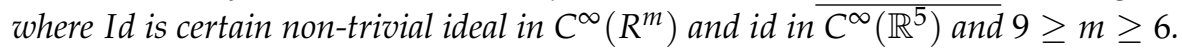

Proof. Every small exotic $R^{4}$ is embeddable smoothly as an open subset of $\mathbb{R}^{4}$ so the result for small $R^{4}$ follows from [13] (Corollary 2.2, p. 25). Large $R^{4 \prime}$ s are not embeddable in $\mathbb{R}^{4}$ but are still smooth manifolds so they are representable in the claimed form, i.e., $C^{\infty}\left(R^{4}\right)$ is finitely presented $C^{\infty}\left(R^{4}\right)=C^{\infty}\left(\mathbb{R}^{m}\right) / I d$ where $I d$ is finitely generated [13] (p. 24). The upper bound $m \leq 9$ follows from the Whitney embedding theorem for the real smooth manifolds.

Remark 7. It would be interesting to give the explicit description of ideals distinguishing between different exotic $R^{4}$ 's. Such task presumably requires making use of complicated boundaries of Casson handless which are 
infinite geometric constructions. As far as we know such an approach to invariants of $R^{4}$ 's have never been carried out.

Here we will follow different strategy without direct referring to Casson handles. We are developing new kind of symmetry for exotic smoothness structures on $\mathbb{R}^{4}$ based on $\mathcal{B}$ and Set.

Let us consider a smooth manifold $M$ in Set, $M \in \mathbb{M}$, with a smooth atlas $\left\{\left(U_{\alpha}, \phi_{\alpha}\right)\right\}_{\alpha \in I}$. We assign to each $\alpha \in I$ a map $s: \mathbb{M} \rightarrow \mathcal{B}, s: U_{\alpha} \rightarrow s\left(U_{\alpha}\right)$ or the identity (isomorphism in Set, diffeomorphism) $i: U_{\alpha} \rightarrow U_{\alpha}$ in Set. Thus, the following function is defined

$$
\forall(\alpha \in I) U_{\alpha} \mapsto \begin{cases}i\left(U_{\alpha}\right) \simeq U_{\alpha}, & \text { for } \alpha \in A \subset I \\ s\left(U_{\alpha}\right) \in \mathcal{B}, & \text { for } \alpha \in I \backslash A\end{cases}
$$

Definition 1. (a) $\mathcal{B}$-cover of a smooth manifold $M$ is the above assignment (6) such that $A \neq \varnothing$ and $A \neq I$, i.e.,

$$
\exists(\alpha, \beta \in I) U_{\alpha} \mapsto U_{\alpha} \text { and } U_{\beta} \mapsto s\left(U_{\beta}\right) .
$$

(b) A $\mathcal{B}$-local smooth manifold is a smooth manifold $M$ which every atlas has underlying $\mathcal{B}$-cover, i.e.

$$
\forall(\mathcal{U} \text { an open good cover of } M) \exists(\mathcal{B} \text {-cover defined from } \mathcal{U}) .
$$

Remark 8. When $M$ is certain smooth manifold in Set then to be $\mathcal{B}$-local means that for any its smooth cover $\mathcal{U}=\left(M,\left\{U_{\alpha}\right\}_{\alpha \in I}\right)$ there exists certain $\mathcal{B}$-cover such that every $U_{\alpha} \in \mathcal{U}$ is $i\left(U_{\alpha}\right)$ or $\Gamma U_{\alpha}^{\mathcal{B}}$. We say that a smooth cover of $M$ derives from a $\mathcal{B}$-cover if the smooth cover has underlying $\mathcal{B}$-local atlas.

We are going to explore what structures on $M$ are determined by its $\mathcal{B}$-local covers. First, let us assume that the structure we are looking for is invariant with respect to the choice of the local maps consisting $\mathcal{B}$-covers. Moreover, we want the structure $\widehat{M}$ be a smooth structure of a manifold of the same dimension.

Proposition 2. If $M=\mathbb{R}^{n}$ and $\widehat{M}$ is smooth, then $\widehat{M} \simeq$ exotic $\mathbb{R}^{4}$.

Proof. The result is a direct consequence of the following fundamental fact. Any smooth atlas of any exotic $R^{4}$ cannot contain just one local chart. Moreover, if each smooth atlas on $\mathbb{R}^{4}$ is not a singleelement one such $\mathbb{R}^{4}$ is diffeomorphic to certain exotic $R^{4}$ and is not diffeomorphic to the standard smooth $\mathbb{R}^{4}$. This is simply the consequence of the fact that given a single-element atlas on $\mathbb{R}^{4}$ it is just the standard $\mathbb{R}^{4}$.

Thus, the existence of a $\mathcal{B}$-covers for every open cover of $M$ can change its smoothness structure. We will see in Section 2.2.3 that indeed one finds an action on smooth functions on $M$ in Set which derives from existence of $\mathcal{B}$-covers (again for $M \simeq \mathbb{R}^{4}$ ). This is Definition 4 which makes precise the equivalence of functions modified by $\mathcal{B}$-covers on $\mathbb{R}^{4}$ with functions from certain smoothness structure on $\mathbb{R}^{4}$ in Set. This smoothness structure is called Set-invariant $\mathcal{B}$-local smoothness structure in Section 2.2.3.

Here we use phrase $\mathcal{B}$-invariant smoothness structure or $\mathcal{B}$-invariant structure for indicating this possibility that $\mathcal{B}$-covers can modify smoothness structures on manifolds. The resulting structure is then called $\mathcal{B}$-invariant structure.

Corollary 1. Any realization of the $\mathcal{B}$-invariant smooth structure on any $\widehat{\mathbb{R}^{n}}$ must be an exotic $\mathbb{R}^{4}$.

\subsection{Is Any Exotic $\mathbb{R}^{4}$ Equivalent to a $\mathcal{B}$-Invariant Structure?}

We showed that any $\mathcal{B}$-invariant smooth structure on $\mathbb{R}^{4}$ is an exotic $\mathbb{R}^{4}$. Now we are going to approach the reverse problem: Given any exotic $\mathbb{R}^{4}$ is it generated by the canonical $\mathbb{B}$-invariant smooth 
structure? If Yes and if there are tools to distinguish different exotic structures in terms of $\mathcal{B}$-invariance, which would mean the equivalence of both constructions. We do not resolve this problem here with all generality. Instead we show that $\mathcal{B}$-covers lead us precisely towards understanding of exotic smooth functions on $\mathbb{R}^{4}$. Thus, we will work rather with real-valued functions on $\mathbb{R}^{4}$ than with handle-bodies underlying known exotic $\mathbb{R}^{4}$. Moreover, the $\mathcal{B}$-invariance can be used also for describing exotic smoothings of $\mathbb{R}^{4}$ with finitely many maps in the smooth atlas. The similar approach, though based more on the structure of Casson handles, was initialized in $[17,18]$ and further developed in [19].

Let $R^{4}$ be an exotic $\mathbb{R}^{4}$ when working in Set. When switching to $\mathcal{B} R^{4}$ is the canonical image of $\mathbb{R}^{4}$ via the embedding $s: \mathbb{M} \rightarrow \mathcal{B}$. The meaning of $R^{4}$ will be clear from the context. Given exotic $R^{4}$ in Set we have the space of exotic smooth functions on it $C^{\infty}\left(R^{4}\right)$. The space of standard smooth functions $C^{\infty}\left(\mathbb{R}^{4}\right)$ is related with $C^{\infty}\left(R^{4}\right)$. All exotic and all standard smooth functions are continuous

$$
C^{\infty}\left(R^{4}\right) \subset C^{0}\left(\mathbb{R}^{4}\right) \supset C^{\infty}\left(\mathbb{R}^{4}\right) .
$$

Moreover, if $C^{\infty}\left(\mathbb{R}^{4}\right)=C^{\infty}\left(R^{4}\right)$ then $\mathbb{R}^{4} \stackrel{\text { diff. }}{=} R^{4}[23]$ so for any exotic $R^{4}$ there have to exist smooth exotic functions which are merely continuous with respect to the standard smoothness structure.. Let $f \in C^{0}\left(\mathbb{R}^{4}\right)$ be one of such continuous function which become exotic smooth in some exotic smoothness structure of an exotic $R^{4}$. The subsequent task is to understand and perform infinite many differentiations of this continuous function in the exotic smoothness structure. This kind of problem has been successfully resolved in the theory of distributions. Thus, the question we want to explore further is whether distribution theory can help understanding exotic smoothness on $\mathbb{R}^{4}$.

\subsubsection{First Comparison of Distributions and Exotic Smoothness Structures on $\mathbb{R}^{4}$}

Let us consider a smooth function $f \in C^{\infty}\left(\mathbb{R}^{4}\right)$ and a test function $\phi \in C_{0}^{\infty}\left(\mathbb{R}^{4}\right)=D\left(\mathbb{R}^{4}\right)$ with the support in a certain ball in $\mathbb{R}^{4}$, hence vanishing at infinity. The space $D\left(\mathbb{R}^{4}\right)$ of test functions is the sum of subspaces $D_{K}$, of test functions with supports in $K$, over all compact subsets $K \subset \mathbb{R}^{4}$ [24] (p. 151). Then it holds

$$
\int_{\mathbb{R}^{4}} \partial^{\alpha} f(x) \cdot \phi(x) d x=(-1)^{|\alpha|} \int_{\mathbb{R}^{4}} f(x) \cdot \partial^{\alpha} \phi(x) d x, \text { for all test functions } \phi \in D\left(\mathbb{R}^{4}\right)
$$

where $\alpha=\left(\alpha_{1}, \ldots, \alpha_{4}\right)$ is a multi-index and $|\alpha|=\alpha_{1}+\ldots+\alpha_{4}$ so that $\partial^{\alpha}=\frac{\partial^{\alpha_{1}}}{\partial x_{1}^{\alpha_{1}}} \cdot \ldots \cdot \frac{\partial^{\alpha_{4}}}{\partial x_{4}^{\alpha_{4}}}$. This formula, as is well known, serves as a prescription how to extend the differentiation over nondifferentiable functions from $f \in C^{0}\left(\mathbb{R}^{4}\right)$. Then the rich distribution theory has been developed which extends differentiation over non-continuous functions and over proper distributions not-being functions at all.

Remark 9. The important fact which follows from (7) is that whenever a distribution $h \in D^{\prime}\left(\mathbb{R}^{4}\right)$ is represented by a smooth function $H \in C^{\infty}\left(\mathbb{R}^{4}\right)$ then the distributional differentiation coincides with the usual differentiation of the smooth function $H$ and we write $h=H$ for such regular distributions. Thus, differentiation of continuous functions by distributions preserves differentiations of smooth functions.

Remark 10. This extension of continuous functions is minimal: One must take the space of all distributions to include all continuous functions with all partial derivatives. If we take any proper subclass of distributions then it follows that not all continuous functions were included. This is the consequence of the two following theorems [24] (pp. 167, 169, Theorems. 6.26, 6.28).

Let $\mathcal{D}^{\prime}(\Omega)$ be the space of distributions on open subset $\Omega \subseteq \mathbb{R}^{4}$.

Theorem 1 ([24]). Suppose $h \in \mathcal{D}^{\prime}(\Omega)$, and $K$ is a compact subset of $\Omega$. Then there is a continuous function $f$ in $\Omega$ and there is a multi-index $\alpha$ such that

$$
h \phi=(-1)^{|\alpha|} \int_{\Omega} f(x)\left(D^{\alpha} \phi\right)(x) d x
$$


for every $\phi \in \mathcal{D}_{K}$.

This can be made more global ([24] (p. 169, Th. 6.28).

Theorem 2 ([24]). Suppose $h \in \mathcal{D}^{\prime}(\Omega)$. There exist continuous functions $g_{\alpha}$ in $\Omega$, one for each multi-index $\alpha$, such that

a. each compact $K \subset \Omega$ intersects the supports of only finitely many $g_{\alpha}$, and

b. $h=\sum_{\alpha} D^{\alpha} g_{\alpha}$

If $h$ has finite order, then the functions $g_{\alpha}$ can be chosen so that only finitely many are different from 0.

Let us note that quite analogous situation takes place for exotic smooth functions on $\mathbb{R}^{4}$. As we observed before given an exotic $R^{4}$ some continuous functions become differentiable and some standard smooth become nondifferentiable but only continuous. To distinguish one exotic smoothness structure from another, one should find a way to distillate the set of continuous functions which become exotic smooth when switching between various $R^{4 \prime}$ s. This is currently out of our reach (however, see [25] where functional subspaces were analyzed from the point of view of trees defining Casson handless).

\subsubsection{Distributions in $\mathcal{B}$}

Distribution theory in the topos $\mathcal{B}$ has been presented in [13]. The choice of the topos $\mathcal{B}$ is crucial here. As we noted already in Remark 1 the canonical NNO in $\mathcal{B}$ is the constant sheaf $\underline{\mathbb{N}}=\mathbb{N}$ which, however, leads to the variety of problems regarding smooth manifolds in $\mathcal{B}$. The crucial for curing these weaknesses and for the proper representation of distributions in $\mathcal{B}$ is to substitute $\mathbb{N}$ by another NNO object. The substitution is the object of smooth natural numbers, $N$, which is a kind of intuitionistic end-extension of $\mathbb{N}$ in $\mathcal{B}$. Such consistent replacement is one of the main topics of [13].

Remark 11. The object $N$ contains nonstandard big natural numbers [13] and $R$ either. Because of that $R=C^{\infty}(\mathbb{R})$ is non-Archimedean with respect to $\mathbb{N}$ and becomes Archimedean with respect to $N$. Moreover, problems stated in (2) are now cured because of referring to $N$

- $\quad s: \mathbb{M} \rightarrow \mathcal{B}$ preserve compactness, e.g., $[0,1] \subset R=s(\mathbb{R})$ is s-compact (smooth compact, i.e., with respect to $N$ );

- $\quad$ s preserves open covers with respect to $N$;

- $\quad$ s preserves partitions of unity subordinated to open covers (with respect to $N$ );

- the ring $R$ is a local ring;

Let us follow the description of internal to $\mathcal{B}$ distributions. As we mentioned above $R$ is a non-Archimedean ring with respect to $\mathbb{N}$ which becomes Archimedean relating to $N$. The reason is that $N$ contains infinite big natural numbers $r \in N \backslash \mathbb{N}$ which, however, can be inverted, i.e., $\frac{1}{r} \in R$. Thus, one has the object of invertible infinitesimals $\mathbb{I}$ as sub-object of $R$. Since $\mathcal{B}$ is a model of synthetic differential geometry there exists also the object $\triangle$ of non-invertible infinitesimals. More precisely, let $\square=\left\{x \in R: \forall_{n \in \mathbb{N}}-\frac{1}{n+1}<x<\frac{1}{n+1}\right\}$ be a space (sub-object of $R$ in $\mathcal{B}$ ) of infinitesimals. There are two sub-objects of $\square$

$$
\begin{gathered}
\mathbb{I}=\{x \in \square: x \text { is invertible }\}=\left\{x \in \square: \exists_{n \in N} x<\frac{1}{n+1} \text { or } x>\frac{-1}{n+1}\right\} \\
\triangle=\{x \in R: x \text { is not invertible }\}=\left\{x \in R: \forall_{n \in N}-\frac{1}{n+1}<x<\frac{1}{n+1}\right\} .
\end{gathered}
$$

The ring of accessible reals is defined as

$$
R_{\mathrm{acc}}=\left\{x \in R: \exists_{n \in \mathbb{N}}-n<x<n\right\} .
$$


Remark 12. Note the appearance of different types, $\mathbb{N}$ and $N$, in definitions above. Thinking in Set the accessible reals comprise those which are Archimedean with respect to $\mathbb{N}$ - the standard natural numbers. Infinitesimals i.e., the object $\square$ can be well presented in terms of the apartness relation \# on $R: x \# y$ iff $\exists_{n \in \mathbb{N}}|x-y|>\frac{1}{n}$, then

$$
\square=\{x \in R: \neg(x \# 0)\} .
$$

So any infinitesimal cannot be apparted from zero by any $\frac{1}{n}, n \in \mathbb{N}$.

Remark 13. Internal logic of toposes is intuitionistic logic and because of that there exist non-invertible infinitesimals in $\mathcal{B}$ which cannot exist in Set.

A function $f \in R^{R^{n}}$ is accessible if for every multi-index $\alpha$ and $x \in R_{\text {acc }}, \partial^{\alpha} f(x) \in R_{\text {acc }}$. $f \in R^{R^{n}}$ has accessible support when $\exists_{m \in \mathbb{N}} \forall_{x \in R^{n}} x \in[-m, m]^{n} \subset R^{n}$ or $f(x)=0$.

Definition 2. A test function $f \in R^{R^{n}}$ is an accessible function with accessible support.

Let $F_{n}$ be the space of all test functions from $R^{R^{n}}$ and $\left(R^{R^{n}}\right)_{a}$ be an object of functions with accessible supports.

Definition 3. A distribution on $R^{n}$ is an $R$-linear map

$$
\mu:\left(R^{R^{n}}\right)_{a} \rightarrow R
$$

which for $f \in\left(R^{R^{n}}\right)_{a}$ fulfils

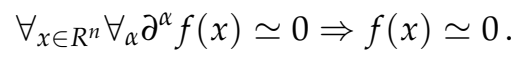

There is direct $1: 1$ correspondence between distributions from $\mathcal{B}$ and Set [13] (p. 336, Th. 3.15.3).

Theorem 3 ([13]). The global sections functor $\Gamma: \mathcal{B} \rightarrow$ Sets induces a bijection between distributions $F_{n} \stackrel{v}{\rightarrow} R$ in $\mathcal{B}$ and external distributions $\Gamma v: C_{c}^{\infty}\left(\mathbb{R}^{n}\right) \rightarrow \mathbb{R}$, and between distributions with compact support, i.e., $R$-linear maps $R^{R^{n}} \stackrel{v}{\rightarrow} R$ and external distribution with compact support $C^{\infty}\left(\mathbb{R}^{n}\right) \stackrel{\Gamma v}{\rightarrow} \mathbb{R}$.

Moreover, every distribution $\mu$ in $\mathcal{B}$ can be represented by a function (predistribution) $\mu_{0}: R_{\mathrm{acc}}^{n} \rightarrow$ $R$. Specifically, every function $\mu_{0}$ as above defines an $R$-linear functional by integration in $\mathcal{B}$

$$
h_{\mu_{0}}(f)=\int \mu_{0} f
$$

If $\mu_{0}$ fulfils the continuity rule

$$
\forall_{f \in F_{n}} \forall_{\alpha \in \mathbb{N}^{n}} \forall_{x \in R} \partial^{\alpha} f(x) \simeq 0 \Rightarrow h_{\mu_{0}}(f) \simeq 0
$$

it is called a predistribution.

Theorem 4 ([13], p. 324, Th. 3.6). For every distribution $\mu$ on $R^{n}$ there exists a predistribution $\mu_{0}: R_{a c c}^{n} \rightarrow R$ such that for all $f \in F^{n}$

$$
\mu(f)=\int f(x) \mu_{0}(x) d x .
$$

Thus, given the $1: 1$ correspondence between external and internal to $\mathcal{B}$ distributions now we have the possibility to represent internally every distribution from Set by regular $\mathcal{B}$-internal distribution.

Remark 14. From the proofs of both theorems above in [13] it follows that the facts stated are true only because of the existence of invertible infinitesimals and replacing $\mathbb{N}$ by $N$ consistently in $\mathcal{B}$. 


\subsubsection{The Construction of $\mathcal{B}$-Invariant Functions as Smooth Exotic}

Given a smooth $\mathcal{B}$-local atlas on $M$ (as in Definition 1), it always contains some local map(s) in $\mathcal{B}$ (see Remark 8) such that some smooth transition function $\phi_{\alpha \beta}: U_{\alpha \beta} \rightarrow U_{\alpha \beta}$ should be replaced by $\phi_{\rightarrow \mathcal{B}}: U_{\alpha \beta} \rightarrow U_{\alpha \beta}^{\mathcal{B}}$. How can we define the transitions from Set to $\mathcal{B}$ and back? Certainly, the obvious choice is to use global section functor $\Gamma: \mathcal{B} \rightarrow$ Set and its left adjoint the constant sheaf functor $\Delta:$ Set $\rightarrow \mathcal{B}$. The pair $(\Gamma, \Delta)$ is a geometric morphism. Additionally, on manifolds $M \in \mathbb{M}$ there exists full and faithful embedding $s: \mathbb{M} \rightarrow \mathcal{B}$ such that $\Gamma(s(M)) \simeq M$ where $\simeq$ is a diffeomorphism of $M$ in Set (see (5)). Thus, given $U_{\alpha \beta}=U_{\alpha} \cap U_{\beta} \simeq \mathbb{R}^{4}$ we can embed it by $s$ into $\mathcal{B}$ and $s\left(U_{\alpha \beta}\right) \simeq R^{4}$.

Now given a continuous function $f \in C^{0}\left(\mathbb{R}^{4}\right), f \notin C^{1}\left(\mathbb{R}^{4}\right)$, we are going to understand it as differentiable or smooth in an exotic smoothness structure on $\mathbb{R}^{4}$. The crucial ingredient of the approach is the allowed local shift $\phi_{\rightarrow \mathcal{B}}$ in the $\mathcal{B}$-atlas.

Definition 4. $\mathcal{B}$-local smoothness structure $\mathcal{U}^{\mathcal{B}}$ on $\mathbb{R}^{4}$ is smoothly equivalent to certain smoothness structure $\mathcal{U}$ on $\mathbb{R}^{4}$ iff every smooth $\mathcal{B}$ function is also smooth in the structure $\mathcal{U}$.

$\mathcal{B}$-local smoothness structure on $\mathbb{R}^{4}$ is Set-invariant when it is smoothly equivalent to certain smoothness structure of $\mathbb{R}^{4}$ in Set.

Remark 15. This definition claims that certain continuous functions which are going to be smoothed out by means of $\mathcal{B}$-local structures can be smoothed out by the change of smoothness structure on $\mathbb{R}^{4}$.

Theorem 5. If one begins with a local smooth cover of $\mathbb{R}^{4}$ and make it $\mathcal{B}$-local smooth by taking some of its local maps as $\mathcal{B}$-local, then Set-invariant smoothness structure equivalent to this $\mathcal{B}$-structure, is not diffeomorphic to the initial one.

To prove this result, we need the following observation.

Proposition 3. For every $\mathcal{B}$-local smooth cover of $\mathbb{R}^{4}$ which is Set-invariant there exist continuous functions in $C^{0}\left(\mathbb{R}^{4}\right)$ which become smooth.

Proof. Let $f \in C^{0}\left(\mathbb{R}^{4}\right)$ be nondifferentiable. We still differentiate it as distribution $C^{0}\left(\mathbb{R}^{4}\right) \ni f \stackrel{\partial}{\longrightarrow}$ $\hat{f} \in D^{\prime}(\Omega), \Omega \subset \mathbb{R}^{4}$. Then the local transition function $\phi_{\rightarrow \mathcal{B}}:$ Set $\rightarrow \mathcal{B}$ sends $\mathbb{R}^{4}$ to $R^{4}$ via $s: \mathbb{M} \rightarrow \mathcal{B}$. The global section functor $\Gamma: \mathcal{B} \rightarrow$ Set determines $1: 1$ correspondence between distributions in $\mathcal{B}$ and external distributions in Set (Theorem 3). This correspondence goes as follows [13] (p. 234). Given a distribution $\hat{f}$ in Set it defines a natural transformation in $\mathcal{B}$ which is the internal $\mathcal{B}$-distribution $\mu_{\hat{f}}$. This natural transformation $\mu_{\hat{f}}$ is defined by components. Let $F(x, y)$ be an element $F \in R^{R^{4}}(\ell A)$ and $A$ is a smooth ring $C^{\infty}\left(\mathbb{R}^{d}\right) / I d$ with $I d$ an ideal in $C^{\infty}\left(\mathbb{R}^{d}\right)$. So $\ell A \in \mathbb{B}$ are stages-components. Given a sheaf in $\mathcal{B}$ e.g., $R$ or $R^{R^{4}}$ one can consider them on stages $\ell A$ and obtain $R(\ell A), R^{R^{4}}(\ell A)$ correspondingly. Also any natural transformation, e.g., $\mu_{\hat{f}}$, is to be defined by components i.e., on $\ell A$ and then it reads $\mu_{\hat{f} \mid \ell A}$. Thus, the definition of $\mu_{\hat{f}}$ on components is as follows (here Id is an ideal in $\left.C^{\infty}\left(\mathbb{R}^{d}\right)\right)$

$$
\begin{array}{r}
\mu_{\hat{f} \mid \ell A}: R^{R^{4}}(\ell A) \rightarrow R(\ell A), A=C^{\infty}\left(\mathbb{R}^{d}\right) / I d . \\
\mu_{\hat{f} \mid \ell A}(F(x, y))=\hat{f}_{y}(F(x, y)), F(-,-): \mathbb{R}^{d} \times \mathbb{R}^{4} \rightarrow \mathbb{R} .
\end{array}
$$

Given an external distribution $\hat{f}$ we have the unique $R$-linear map $\mu_{\hat{f}}$ in $\mathcal{B}$ which is the internal distribution in $\mathcal{B}$. This internal distribution can be marked as $s(\hat{f})$ which indicates the reverse direction of the correspondence, i.e., the global section functor uniquely retrieves the external distribution $\Gamma s(\hat{f})=\hat{f}$. 
The change of the local map from Set to $\mathcal{B}$ sends $\mathbb{R}^{4}$ to $R^{4}$ and $\hat{f}$ to $\mu_{\hat{f}}$. Next step is to represents $\mu_{\hat{f}}$ by the predistribution $\mu_{\hat{f}}^{0}$ such that

$$
\mu_{\hat{f}}^{0} \in R^{R^{4}} \text { and } \mu_{\hat{f}}(g)=\int \mu_{\hat{f}}^{0}(x) \cdot g(x) d x .
$$

$\mu_{\hat{f}}^{0}$ is continuous [13] (Remark 3.9.2, p. 311). Moreover, there always exist its differentials of arbitrarily order (for any 4-index $\alpha$ ) as follows from the Kock axiom [13] (p. 303). Thus, the local change of the map into $\mathcal{B}$-internal defines, in terms of $\mathcal{B}$, smoothness of continuous functions on $\mathbb{R}^{4}$ in Set.

Externally, distributions in Set obtained by differentiating the continuous functions can be represented by continuous functions due to Theorems 1 and 2. More precisely, there exist distributions e.g., from $D^{\prime}(\Omega)$ with respect to the test functions $g \in D_{K}$ as in Theorem 1 which are entirely determined by a continuous functions on $\Omega \subset \mathbb{R}^{4}$.

The diagram below shows the steps performed in the proof above (See Figure 1).

$$
\begin{aligned}
& \phi_{\alpha \beta}: U_{\alpha \beta} \longrightarrow U_{\alpha \beta}: \\
& C^{0}\left(\mathbb{R}^{4}\right) \ni f \stackrel{\partial}{\rightarrow} \hat{f} \in D^{\prime}\left(\mathbb{R}^{4}\right): \\
& 1: 1 \downarrow \text { Th. } 3 \\
& \mu_{f}: R^{R^{4}} \rightarrow R: \\
& 1: 1 \downarrow \text { Th. } 4 \\
& \mu_{f}^{0}: R^{n} \rightarrow R:
\end{aligned}
$$

Figure 1. $\mathcal{B}$-smoothing of the continuous function $f$. Differentiating of $f$ in Set gives rise to the distribution which internally is certain unique $\mathcal{B}$-distribution $\mu_{\hat{f}}$. This last is internally a regular distribution (function) $\mu_{\hat{f}}^{0}$ due to the replacement of $\mathbb{N}$ by $N$.

Proof of Theorem 5. Given a local map from a smooth atlas on $\mathbb{R}^{4}$ let it be changed into $\mathcal{B}$-internal one. It is then possible to find a continuous function on $U_{\alpha} \simeq \mathbb{R}^{4}$ which becomes smooth in the $U_{\alpha}^{\mathcal{B}} \simeq R^{4}$ just by repeating the construction from the proof of Proposition 3. Then the Set-invariant $\mathcal{B}$-smoothness structure on $\mathbb{R}^{4}$ is not be diffeomorphic to the initial smoothness structure.

Remark 16. From the construction above it follows that, in principle, one can make locally any continuous function $f_{\mid U_{\alpha}} \mathcal{B}$-smooth. To distinguish between smoothness structures on $\mathbb{R}^{4}$ we need some additional global information. This is analogous to exotic smooth manifolds $R^{4}$ which locally are describable by standard smooth transition functions. The difference between them is written in some topological information given by Casson handles.

Remark 17. The construction of Theorem 5 can be repeated however at this stage of development of the approach we do not know whether obtained smoothness structures are pairwise different and nondiffeomorphic.

\subsection{Local $\mathcal{B}$-Invariance and General Tovariance}

There is, however, a price to pay for the change $\mathbb{N} \rightarrow N$ in $\mathcal{B}$ : The object of smooth natural numbers $N$ is not preserved by geometric morphisms between topoi ( $\mathbb{N}$ does) - it does not even exist in general. The consequence would be leaving the class of geometric morphisms and geometric theories when approaching exotic 4 -smoothness by $\mathcal{B}$-means.

Remark 18. A geometric morphism $g: \mathcal{T}_{1} \rightarrow \mathcal{T}_{2}$ between toposes $\mathcal{T}_{1}, \mathcal{T}_{2}$ is the pair $\left(g_{*}, g^{*}\right)$ of functors $g_{*}: \mathcal{T}_{1} \rightarrow \mathcal{T}_{2}$ and its left adjoint $g^{*}: \mathcal{T}_{2} \rightarrow \mathcal{T}_{1}$ which preserves finite limits. 
Remark 19. Let $L$ be the 1st order language. The set of geometric formulae in $L$ is the smallest set of formulae containing atomic formulae and closed by finite conjunction $(\wedge)$, arbitrary disjunction $(\vee)$, and existential quantification $(\exists)$.

A theory is geometric if it can be axiomatized by sentences of the form $\forall x(\phi(\vec{x}) \rightarrow \psi(\vec{x}))$ where $\phi$ and $\psi$ are geometric formulae and $\vec{x}=\left(x_{1}, \ldots, x_{n}\right)$ for some $n \in \mathbb{N}$.

Proposition 4 ([11]). The inverse image part $g^{*}$ of a geometric morphism $g: \mathcal{T}_{1} \rightarrow \mathcal{T}_{2}$ preserves any geometric theory.

If $\mathcal{T}$ is a sheaf topos over a site $C$ with $\mathbb{N}$ the object $\mathrm{NNO}$ (e.g., $\mathcal{B}$ with $C \simeq \mathbb{B}$ ), then there is a unique geometric morphism $g=(\Delta \dashv \Gamma): \mathcal{T} \stackrel{\frac{\Delta}{\Gamma}}{\stackrel{\Delta}{r}}$ Set where $\Gamma$ is the global section functor and $\Delta$ the inverse image being the locally constant sheaf functor $(\Delta$ is the sheafification functor of the constant presheaf $\mathbb{N}$ in $\operatorname{Set}^{C p}$ ). Then

$$
\Delta(\mathbb{N})=\Delta\left(\sum_{n \in \mathbb{N}} 1_{\text {Set }}\right)=\sum_{n \in \mathbb{N}}\left(\Delta 1_{\text {Set }}\right) .
$$

Proposition 5 ([22], Lemma A.4.1.14). For any geometric morphism $g=\left(g_{*}, g^{*}\right)$ between toposes $\mathcal{T}_{1}, \mathcal{T}_{2}$ the inverse image functor $g^{*}$ preserves $N N O$, i.e., if $\mathbb{N}_{2}$ is a NNO in $\mathcal{T}_{2}$ then $g^{*} \mathbb{N}_{2}$ is the $N N O$ in $\mathcal{T}_{1}$.

It follows

Corollary 2. The object $N$ of smooth natural numbers in $\mathcal{B}$ is not preserved by the geometric morphism $g=(\Delta \dashv \Gamma)$ between $\mathcal{B}$ and Set.

Proof. $N$ is not isomorphic to $\mathbb{N}_{\mathcal{B}}$ in $\mathcal{B}$ and for the geometric morphism $g: \mathcal{B} \rightarrow$ Set the inverse image part of it, $g^{*}$, sends $\mathbb{N}_{\text {Set }}$ to $\mathbb{N}_{\mathcal{B}} \neq N$.

Similarly, it holds

Corollary 3. Given a topos $\mathcal{T}$ with $N N O$ and a geometric morphism $g=\left(g_{*}, g^{*}\right): \mathcal{B} \rightarrow \mathcal{T}$, then $N$ in $\mathcal{B}$ is not preserved by g, i.e., $g^{*} \mathbb{N}_{\mathcal{T}} \neq N$.

Remark 20. $N$ is not NNO in $\mathcal{B}$. The arithmetic of natural numbers based on $N$ is a weaker form of the arithmetic based on $\mathbb{N}$ e.g., coherent induction principle holds instead of induction [13]. However, the distinction between $N$ and $\mathbb{N}$ in $\mathcal{B}$ remains undecidable and hard to be grasped [13] ( $p$. 307).

Let $(\mathcal{B}, \mathbb{N}),(\mathcal{B}, N)$ be the topos $\mathcal{B}$ with the canonical $N N O, \mathbb{N}$, and with $N$ the object of smooth natural numbers, respectively. Let the meta-procedure of consistent replacement of $\mathbb{N}$ by $N$ be abbreviated as $\delta_{\mathcal{B}}$. Hence $\delta_{\mathcal{B}}:(\mathcal{B}, \mathbb{N}) \rightarrow(\mathcal{B}, N)$. In particular, $\delta_{\mathcal{B}} \mathbb{N} \simeq N, \delta_{\mathcal{B}} R_{\mathbb{N}} \simeq R_{N}$ and $R_{\mathbb{N}}$ is non-Archimedean, nonlocal ring in $\mathcal{B}$ while $R_{N}$ is Archimedean and local ring. The best way to describe $\delta_{\mathcal{B}}$ is via a corresponding shift in axiomatic systems [13]. Such a shift leads to the weakening of arithmetic. However, as we saw in Corollaries above, smooth $N$ replacing the canonical NNO might be a source of non-geometricity in theories formulated in $(\mathcal{B}, N)$ which are based on $N$.

For example, the theory of distributions in $(\mathcal{B}, N)$ and the meta correspondence as in Theorem 3 are non-geometric since they rely essentially on smooth natural numbers $N$. If these results have additionally physical implications that would be a way showing the breaking of the tovariance principle [11]. Below we will show how much we can push forward this program based on the results of this paper.

The original general tovariance principle as in [11] relies on the free choice of arbitrary topos $\mathcal{T}$ with NNO such that any physical quantum theory formulated in the geometric language internally to $\mathcal{T}$ is equivalent to any other formulation internal to $\mathcal{T}^{\prime}$, provided the change $g: \mathcal{T} \rightarrow \mathcal{T}^{\prime}$ is a geometric morphism. It follows that physics does not distinguish between these two representations 
and hence geometric logic is the language in which physical theories should be formulated. [11]. Consequently, any quantum theory with the quantum algebra of observables is equivalent to certain commutative algebra of a classical theory. The equivalence is in terms of physical indistinguishability which resembles effects of gravity in GR. They are locally indistinguishable from the acceleration of the physical frame by any physical experiment. This quantum-classical equivalence for toposes is a direct consequence of the fact that for each noncommutative $C^{*}$-algebra $\mathcal{C}$ there exists a topos $\mathcal{T}_{\mathcal{C}}$ in which $\mathcal{C}$ becomes internally a commutative algebra. However, already in [11] the authors observed that the construction of a $C^{*}$-algebra is not geometric (the completeness property and the existence of a norm are not geometric in general) and that strong formulation of the tovariance principle may be broken and not giving the strict equivalence of quantum and classical formulations. Consequently the authors themselves quit referring to this principle as general physical rule [12] (p. 493). However, still the point-free version of $C^{*}$-algebras (localic $C^{*}$-algebras) have been developed [26] which are applicable to any topos and thus giving rise to the constructive version of the tovariance principle (We are greatly indebted to the anonymous referee for indicating this fact to us.). We are not making any specific use of the quantum-classical equivalence here, which, anyway, was the main reason for considering general tovariance. Rather we consider a theory, eventually in the geometric language, as physically (and mathematically) invariant with respect to the various interpretations in toposes with NNO. Thus, at this stage, we can restrict the class of physical theories to these theories which are formulated in the geometric language. Even this is done the shift $\delta_{\mathcal{B}}$, replacing $\mathbb{N}$ by $N$, is the source of non-geometricity which cannot be preserved by geometric morphisms. That is why the general tovariance principle gains the following weaker form becoming special tovariance principle

Special tovariance principle. When a physical theory is formulated in the geometric language one cannot experimentally distinguish between their realizations in different toposes with $\mathrm{NNO}$ when the change of the toposes is via geometric morphisms.

We thus left in this formulation undecided the question whether all physical theories can be formulated as geometric theories. If they were (in a sufficiently general form) and if we changed toposes by geometric morphisms then QM in the geometric formulation would lead to $C^{*}$-algebras which be physically equivalent to commutative algebras of observables [11].

Even though this weak form of the tovariance principle were true the approach to exotic smoothness in this paper shows that the original strong general tovariance principle could be broken and the weak form above is too restrictive. Let us indicate two important ingredients of the breaking process.

- We follow 'global-to-local' pattern known from gauge theories. This means that given local $\mathcal{B}$-structure on a smooth manifold $M \in \mathbb{M}$ one switches between Set and $\mathcal{B}$ frames without possibility to leave entirely any of them (Definition 1). The Definition 1 can thus serve as an obstruction to the global choice of a topos on $M$, i.e., Set or $\mathcal{B}$. It can be restated as the property of non-existence of any global Set or $\mathcal{B}$-sections on $M$.

- Any $\mathcal{B}$-invariant structure on $M$ would rely on generalized equivalence between the Set construction with respect to $\mathbb{N}$ and $\mathcal{B}$ construction with respect to $N$. The constructions are not equivalent by geometric morphisms since $N$ is not preserved - it does not exist in general (Corollaries 2 and 3 ).

Thus, even if a theory is geometrically formulated local $\mathcal{B}$-invariance can enforce its nongeometricity and the weak tovariance principle would be too restrictive as allowing only for geometric theories. However, as far as the non-geometric modifications like $\mathbb{N} \rightarrow N$ give rise to no physical implications, the status of the tovariance principle would be still unaffected on this ground.

To decide this problem, we are searching for an exotic $R^{4}$ which has clear physical implications. It is $R^{4}$ embedded in $K 3 \# \overline{C P 2}$ and considered in [15]. It was shown that the curvature of such embedded $R^{4}$ is responsible for the realistic value of the cosmological constant [15] which, moreover, is a topological invariant. This is a really strong connection of a physical quantity and topological structure of this $R^{4}$. If we assume that it is derived as some local $\mathcal{B}$-structure then we can state the following conjecture 
Conjecture 1. If the exotic $R^{4}$ as above is equivalent to certain Set-invariant local $\mathcal{B}$-structure (see Definition 4) then the strong general tovariance principle do not hold true and the weak tovariance principle is too restrictive.

The above conjecture in this formulation is true but still we do not know whether the 'if' assumption can be skipped. We will address this issue elsewhere.

\section{Discussion}

The results of this paper can be seen in a broader categorical perspective. The switching between Set and a topos $\mathcal{B}$ locally on $M \in \mathbb{M}$ corresponds to switching between intuitionistic and 2-valued classical descriptions. As we noted already when an intuitionistic theory is formulated also by geometric formulae and the change between toposes is given by geometric morphisms this precisely defines the invariant theory which is not sensitive to the choice of a topos. On the other side one can reduce the allowed class of toposes such that each of them would determine uniquely the category Set. Then the switching between such toposes and Set is allowed as non-constraining with respect to the (local) Set descriptions. Set is a topos and one can extract categorical properties ensuring that a given topos is equivalent to Set. Crucial property is well-pointedness which enforces that external and internal properties of a well-pointed topos are the same, hence the external/internal symmetry is reduced to the Set-based symmetry. More precisely, let $E$ be a well-pointed topos. By definition it means that $E$ is an elementary topos (defined in 1st order theory) and

- The global section functor $\Gamma: E \rightarrow$ Set is faithful一the terminal object is a generator,

- 1 is not an initial object ( $E$ is nondegenerate).

Theorem 6 ([27]). Set is, up to equivalence, the unique locally small and cocomplete well-pointed topos (or the unique locally small and complete well-pointed topos).

In particular the sub-object classifier $\Omega$ of $E$ has two global elements $\{\perp, \top\}$ and $\Gamma$ in Set is the identity functor. The invariance with respect to such well-pointed and complete toposes does not essentially extends usual invariance in Set.

Then let us extend the allowed class of toposes such that they are not necessarily well-pointed. Again, the restriction to geometric morphisms and intuitionistic geometric languages make the job and an invariance is defined. However, the local on a smooth manifold $M$ topos-based structures work differently. $\mathcal{B}$-local structure on $M$ enforces that a resulting structure is not entirely in Set or $\mathcal{B}$ but still can be equivalent to certain structures in Set. As we noted already the invariance in this case extends geometric components since $N$ replaces $\mathbb{N}$ in $\mathcal{B}$ locally on $M$. Since both Set local chart and $\mathcal{B}$-chart are active on $M$, thus the switch $\mathbb{N} \rightarrow N$ either. From Set perspective one way to grasp this is via suitable weakening of the arithmetic of natural numbers, i.e., Peano arithmetic such that $N$ and $\mathbb{N}$ become interchangeable. As a matter of fact, this kind of task was the guiding principle of the approach in [13] though from the $\mathcal{B}$ perspective. The authors have built the intuitionistic environment, the topos $\mathcal{B}$, where $\mathbb{N}$ is consistently replaced by $N$. They also discussed the suitable weakening of Peano arithmetic allowing for such replacement.

Here we stipulate rather on the local character of the weakening: Depending on the chart of $M$ one has Set with $\mathbb{N}$-based description though in another chart there is $\mathcal{B}$ with $N$-based description. Resulting structure on manifold should be invariant with respect to the choice of such local descriptions. We have not presented entire theory of such structures here. Rather we have focused on the particular construction relating exotic smoothings on $\mathbb{R}^{4}$ which, however, might serve probably as representable for a general theory. We will work on this in a separate publication.

An approach to such general theory were performed by one of the present author in [17] where a rule of indistinguishability of certain models of Peano arithmetic (PA), $\mathbb{N}$ and ${ }^{*} N$, in certain formal environment assigned to a formal theory was proposed. It would be interesting to find a unifying 
formal description of such theory applicable also in the case of this paper. In particular, the role of the models $\left(\mathbb{N},{ }^{*} N\right)$ and their indistinguishability could be played by $(\mathbb{N}, N)$ in our case.

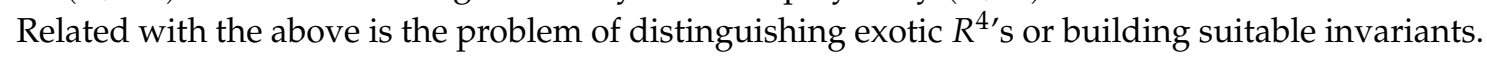
Please note that ${ }^{*} N$ is a nonstandard model of PA build by the ultrafilter construction while $N$ in $B$ is intuitionistic nonstandard model of the weak PA which also contains nonstandard infinite smooth numbers. Thus, switching between Set and $\mathcal{B}$ we switch between ${ }^{*} N$ and $N$ and thus unique $N$ gains infinite many possible Set images. The symmetry of the space of non-principal ultrafilters on $\mathbb{N}$ might

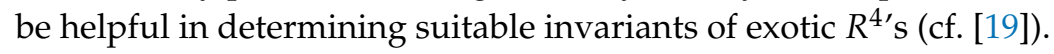

Another important aspect of the approach is the possible use of the presented description of exotic $R^{4}$ to the case of those allowing for the finitely many local charts and consequently can help understanding of the problem of existence of an exotic $S^{4}$. The important feature of our approach is independence on Casson handles constructions (e.g., [16]) hence can be applied to a different class of exotic $R^{4}$ s. Even though the studies are only at the conceptual stage at present, any progress in this field could shed light on the existence of another, unknown so far, smoothings of $\mathbb{R}^{4}$.

The applications to physics can also be worked out. The strict tovariance principle is overpassed by exotic $R^{4 \prime}$ s which are local $\mathcal{B}$-structures as described in this paper. On the other side the applications to

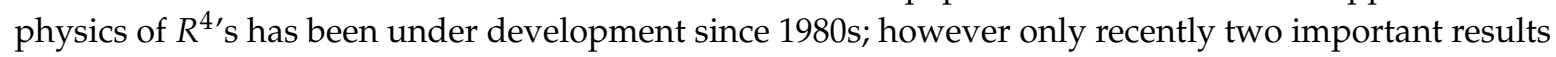
were worked out: Large exotic $R^{4 \prime}$ s are gravitational instantons [14] and the curvature of embedded in $K 3 \# \overline{C P 2}$ exotic $R^{4}$ is a topologically supported realistic value of the cosmological constant [15].

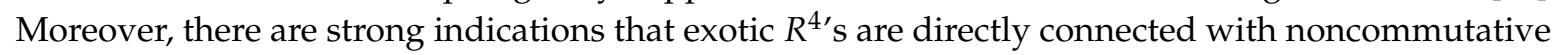
von Neuman algebras [28,29]. In particular, the initial quantum state in the evolution of the universe is geometrically interpreted as wildly embedded 3-sphere and its noncommutative algebra follows from

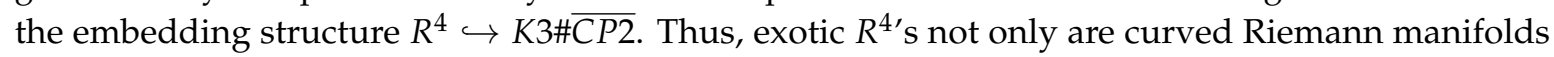
and large are gravitational instantons, but also they determine quantum structures topologically. In the paper we have found and explore the symmetry principle allowing for such results. The symmetry extends usual one defined in Set in such a way that logic and set theory become local physical variables and the invariance with respect to their local changes implies the modification of 4-smoothness on spacetime. If logic were constant (global) no such explanation would be possible (see Corollary 1 and Proposition 2). Moreover, if exotic $R^{4 \prime}$ s are indeed derived as $\mathcal{B}$-local structures (Conjecture 1 ) that would mean that the dynamics of the changes of logic on spacetime gives rise to physical effects such as the explanation of the smallness of the cosmological constant. Then in the search for the final theory of quantum gravity one should deal with such extended symmetry and varying logic. On the other side, bearing in mind the role played by exotic smoothness on open 4-manifolds in determining quantum regime (e.g., [28]) the approach called 'Bohr's doctrines of classical concepts', assigning at final stage classical windows to any quantum theory, found here a 'smooth' ally. Working in this field should certainly increase our understanding of important fundamental aspects of physical problems and indicates the required modifications of the mathematical formalism.

Author Contributions: All authors contributed equally to this work.

Acknowledgments: The authors appreciate much the critical remarks made by referees since all corrections improved the paper substantially. J.K. appreciates the influential discussion with Michael Heller regarding the problems in the paper.

Conflicts of Interest: The authors declare no conflict of interest.

\section{References}

1. Heunen, C.; Landsman, N.P.; Spitters, B. A topos for algebraic quantum theory. Commun. Math. Phys. 2009, 291, 63-110. [CrossRef]

2. Heunen, C.; Landsman, N.P.; Spitters, B. Bohrification of operator algebras and quantum logic. Synthese 2012, 186, 719-752. [CrossRef] 
3. Heunen, C.; Landsman, N.P.; Spitters, B.; Wolters, S. The Gelfand spectrum of a noncommutative C*-algebra: A topos-theoretic approach. J. Aust. Math. Soc. 2012, 90, 32-59. [CrossRef]

4. Isham, C.J.; Butterfield, J. Topos perspective on the Kochen-Specker theorem. I. Quantum states as generalized valuations. Int. J. Theor. Phys. 1998, 37, 2669-2733. [CrossRef]

5. Döoring, A.; Isham, C.J. A topos foundation for theories of physics. I. Formal languages for physics. J. Math. Phys. 2008, 49, 053515. [CrossRef]

6. Döring, A.; Isham, C.J. A topos foundation for theories of physics. II. Daseinisation and the liberation of quantum theory. J. Math. Phys. 2008, 49, 053516. [CrossRef]

7. Döring, A.; Isham, C.J. A topos foundation for theories of physics. III. The representationof physical quantities with arrows. J. Math. Phys. 2008, 49, 053517. [CrossRef]

8. Döring, A.; Isham, C.J. A topos foundation for theories of physics. IV. Categories of systems. J. Math. Phys. 2008, 49, 053518. [CrossRef]

9. Döring, A.; Isham, C.J. “What Is a Thing?": Topos Theory in the Foundations of Physics. Lecture Notes Phys. 2010, 813, 753-937.

10. Bell, J.L. From local to absolute mathematics. Synthese 1986, 69, 409-426. [CrossRef]

11. Heunen, C.; Landsman, N.P.; Spitters, B. The principle of general tovariance. In Proceedings of the Geometry and Physics: XVI International Fall Workshop (Lisboa 2007), Lisboa, Portugal, 5-8 September 2007; pp. $93-102$.

12. Landsman, K. Foundations of Quantum Theory. From Classical Concepts to Operator Algebras; Fundamental Theories of Physics; Springer: Basel, Switzerland, 2017; Volume 188.

13. Moerdijk, I.; Reyes, G.E. Models for Smooth Infinitesimal Analysis; Springer Science + Business Media: New York, NY, USA, 1991.

14. Etesi, G. Strong cosmic censorship and topology change in four dimensional gravity. arXiv 2019, arXiv:1905.03952.

15. Asselmeyer-Maluga, T.; Król, J. How to obtain a cosmological constant from small exotic $\mathbb{R}^{4}$. Phys. Dark Universe 2018, 19, 66-77. [CrossRef]

16. Gompf, R.; Stipsicz, A. 4-Manifolds and Kirby Calculus; AMS: Providence, RI, USA, 1999.

17. Król, J. Background Independence in Quantum Gravity and Forcing Constructions. Found. Phys. 2004, $34,361-403$. [CrossRef]

18. Król, J. Exotic smoothness and noncommutative spaces. The model-teoretical approach. Found. Phys. 2004, 34, 843-861. [CrossRef]

19. Król, J. Model and set-theoretic aspects of smoothness structures on $\mathbb{R}^{4}$. In At the Frontier of Spacetime; Fundamental Theories of Physics; Springer: Basel, Switzerland, 2016; Volume 183; pp. 217-240.

20. Lee, J.M. Introduction to Smooth Manifolds; Springer Science + Business Media: New York, NY, USA, 2006.

21. $n$ Lab, Good Open Cover. Available online: https://ncatlab.org/nlab/show/good+open+cover (accessed on 30 September 2019).

22. Johnstone, P. Sketches of an Elephant: A Topos Theory Compendium; Oxford University Press: Oxford, UK, 2002.

23. StackExchange, Mathematics, Question 1764947. Available online: https://math.stackexchange.com/ questions /1764947 / (accessed on 30 September 2019).

24. Rudin, W. Functional Analysis; McGraw-Hill: Singapore, 1991.

25. Kato, T. ASD moduli spaces over four-manifolds with tree-like ends. Geom. Topol. 2004, 8, 779-830. [CrossRef]

26. Henry, S. Localic Metric spaces and the localic Gelfand duality. arXiv 2014, arXiv:1411.0898.

27. $n$ Lab, Cocomplete Well-Pointed Topos. Available online: https://ncatlab.org/nlab/show/cocomplete+wellpointed+topos (accessed on 25 September 2019).

28. Asselmeyer-Maluga, T. Smooth quantum gravity: Exotic smoothness and quantum gravity. In At the Frontier of Spacetime; Fundamental Theories of Physics; Springer: Basel, Switzerland, 2016; Volume 183, pp. 247-308.

29. Etesi, G. The von Neumann algebra of smooth four-manifolds and a quantum theory of space-time and gravity. arXiv 2017, arXiv:1712.01828.

(C) 2019 by the authors. Licensee MDPI, Basel, Switzerland. This article is an open access article distributed under the terms and conditions of the Creative Commons Attribution (CC BY) license (http://creativecommons.org/licenses/by/4.0/). 